\title{
Image-Oriented Economic Perspective on User Behavior in Multimedia Social Forums: An Analysis on Supply, Consumption, and Saliency
}

\author{
Sangmin $\mathrm{Oh}^{\mathrm{a}, * *}$, Megha Pandey ${ }^{\mathrm{b}}$, Ilseo Kimc ${ }^{\mathrm{c}}$, Anthony Hoogs ${ }^{\mathrm{d}}$ \\ ${ }^{a}$ NVIDIA Corp., 2703 San Tomas Expressway, Santa Clara, CA 95050, U.S.A. \\ ${ }^{b}$ Institute of Infocomm Research, 1 Fusionopolis Way, 138632, Singapore \\ ${ }^{c}$ Amazon.com Inc., 410 Terry Avenue North, Seattle, WA 98109, U.S.A. \\ ${ }^{d}$ Kitware Inc., 28 Corporate Dr., Clifton Park, NY 12065, U.S.A.
}

\begin{abstract}
This work addresses the novel problem of analyzing individual user's behavioral patterns regarding images shared on social forums. In particular, we present an image-oriented economic perspective: the first activity mode of sharing or posting on social forums is interpreted as supply; and another mode of activity such as commenting on images is interpreted as consumption. First, we show that, despite the significant diversity, images in social forums can be clustered into semantically meaningful groups using modern computer vision techniques. Then, users' supply and consumption profiles are characterized based on the distribution of images which they engage with. We then present various statistical analyses on real-world data, which show that there is significant difference between the images users supply and consume. This finding suggests that the flow of images on social network should be modeled as a bi-directional graph. In addition, we introduce a statistical approach to identify users with salient profiles. This approach can be useful for social multimedia services to block users with undesirable behavior or to identify viral content and promote it.
\end{abstract}

(c) 2015 Elsevier Ltd. All rights reserved.

\section{Introduction}

The importance of social multimedia services in our society has been increasing exponentially in recent years as a medium for culture, personal communication, news sharing, and commerce. In particular, diverse images and videos are shared through these services (e.g., Twitter, Flickr, Reddit, Instagram), which are consumed by other users. Such activities are facilitated via social structure (friends, communities etc.), as well as tools such as subscription-based data feeds. Accordingly, with shared goals of making these services more effective, researchers have been making rapid progress in understanding user behavior and content propagation. Notable studies include Cha et al. (2009b,a); Petrovic et al. (2011); Can et al. (2013); Lakkaraju et al. (2013); Uysal and Croft (2011); Sundaram et al. (2012); Danescu-Niculescu-Mizil et al. (2013). Nonetheless,

\footnotetext{
${ }^{* *}$ Corresponding author: Tel.: +1-408-486-4685;

e-mail: sangmino@nvidia.com (Sangmin Oh), pandeym@i2r.a-star.edu.sg (Megha Pandey), ilseo@amazon.com (Ilseo Kim), anthony.hoogs@kitware.com (Anthony Hoogs)
}

most of the existing work focuses on textual data, and very limited study has been reported on user engagement with visual data and how images propagate on online social forums.

In this work $^{1}$, we focus on the novel problem of analyzing individual user's behavioral patterns regarding visual multimedia (i.e., images) shared on social forums. The wide adoption of camera-equipped personal devices has facilitated sharing of images on social media in recent years. Even on Twitter, which is designed for microblogs with short text, a large amount of tweets carry embedded images. For example, our own online collection of Twitter stream shows that approximately 19 percent of tweets carry multimedia data. Hence, the problem of understanding the type of visual content people post and engage with, is becoming more important than ever. To the best of our knowledge, although existing work Cha et al. (2009b,a); Lakkaraju et al. (2013); Sundaram et al. (2012); Can et al. (2013) has studied certain aspects of images and videos on social media, analysis of individual user behavior regarding en-

\footnotetext{
${ }^{1}$ This work was done while the authors were with Kitware Inc.
} 
gagement with visual content has been lacking, and our work provides novel insights in this direction.

In particular, we introduce an image-oriented economic perspective on user activities in social multimedia forums: the first activity mode of sharing or posting is interpreted as 'supply'; another mode of activity such as commenting on images is interpreted as 'consumption'. Analysis of these two modes is important to understand content propagation better, as well as to provide improved personalized services to users. Towards this goal, we have conducted a data-driven analysis of two behavioral modes using the Reddit image dataset Lakkaraju et al. (2013), which contains images shared on reddit.com. Reddit is a popular social forum where people upload posts, with or without images. Other users can comment on, vote up or down on each post. The dataset consists of a total of nearly $17 \mathrm{~K}$ unique images, collected over a timespan of 4.5 years. Additionally, for every image, it contains a metadata log with additional information, which makes it ideal for this research.

Novel contributions of this work include an approach to characterize users' supply and consumption profiles, and a statistical analysis result which showcases the differences between a user's two activity modes. In our approach, two profiles of a user are characterized as a distribution of image categories with which the user interacts. One challenge of analyzing images in social multimedia forums is that they are extremely diverse. For example, Figure 1(a) shows randomly selected images from the Reddit image dataset, which exhibits significant variation. This work shows that even for an in-the-wild dataset such as Reddit image dataset, meaningful image clusters can be obtained based on visual features. Fig. 1(b) shows sample image groups, such as people, text clips, cartoons, and screen shots, obtained by the proposed approach. From these sample image groups, user profiles are computed as bag-of-words (BoW) representations of the categories, effectively capturing the image styles users prefer to supply and consume.

Finally, by comparing users' supply and consumption profiles, as much as $\sim 40 \%$ of users are found to show fairly different supply and consumption patterns. This finding contradicts a baseline hypothesis that users are likely to post and comment on similar image categories, and suggests that personalization of social multimedia services needs to be optimized from both the angles, rather than pursuing a single model per user. Most existing research focuses only on consumption patterns Petrovic et al. (2011); Can et al. (2013); Lakkaraju et al. (2013); Uysal and Croft (2011); Sundaram et al. (2012). To the best of our knowledge, this is the first study to concretely show that there can be significant differences between the two behavioral modes.

As another contribution, we present a statistical approach to identify users with salient profiles, despite the inherent multimodal nature of user bases on social forums. By definition, a salient user exhibits a unique profile. Identifying such users can be beneficial for social media services in multiple ways. For example, such a user may be a trend setter who can potentially create viral content that social media services may want to consider promoting heavily and draw new users. In other cases, such a user may be posting illegal or prohibited content that is outside the norm of the common user base. One difficulty in identifying salient users is that the users of social media services are very diverse. There are multiple communities with different interests, which naturally creates a multi-modal distribution of user profiles. We propose a detection method to identify salient users, which is based on non-parametric kernel density estimation framework Epanechnikov (1969). Finally, we show that certain users' profiles can change dynamically over time and that the proposed method can be extended to be used in such scenarios, providing the ability to identify trending images which contribute the most towards user saliency. We showcase the usefulness of the proposed methods through qualitative experimental results.

The remainder of the paper is organized as follows. In Section 2, related work is reviewed. Section 3 describes the methodologies utilized to characterize individual user's supply and consumption profiles. It also presents our analysis on the similarities and differences between these two behavioral modes. Finally, Section 4 introduces our proposed approaches for salient user detection and key images based on the images supplied by users.

\section{Related Work}

Several researchers such as Hong et al. (2011), Petrovic et al. (2011) and Uysal and Croft (2011) have studied the problem of modeling and predicting the popularity of posts on Twitter. Majority of work in this direction has been focused only on text content of tweets, and analysis of visual content accompanying tweets has been disregarded. In Hong et al. (2011) and Petrovic et al. (2011), the authors studied content propagation to predict retweet counts at the community level. The work of Uysal and Croft (2011) is closer to ours, in that they take a user-centric approach and predict retweet likelihood on an individual basis. However, they, too, focus on text input only.

Some previous works analyze visual content on social forums to understand the propagation of content across social networks. Can et al. (2013) exploited embedded multimedia content in a tweet to predict retweet counts. Notable works also include Cha et al. (2009a) and Cha et al. (2009b) which analyze content posted on Youtube and Flickr, respectively. They learn the patterns of popularity and propagation by taking into account factors such as the amount of time passed since the post was made and the network of the person posting the content. Sundaram et al. (2012) analyzed Flickr and Youtube content to understand the nature of social interactions engendered by visual multimedia. Lakkaraju et al. (2013) introduced the Reddit image dataset, which is also used in this work. They proposed a model to predict the popularity of a post based on several factors, which include the time a post was made, similarity to other viral posts, title etc. They, however, did not analyze the visual content itself, which is the primary focus of this work. Further, all of these studies view the posts at the community level and do not look into the patterns related to individuals. In this work, we take a more user-centric approach and focus on discovering supply and consumption patterns for individuals.

There has also been work which makes use of user-supplied image tags on online photos as a source of data to aid image un- 


\section{(b) Grouping of Images into Multiple Categories using Visual features}

(a) Images from reddit.com
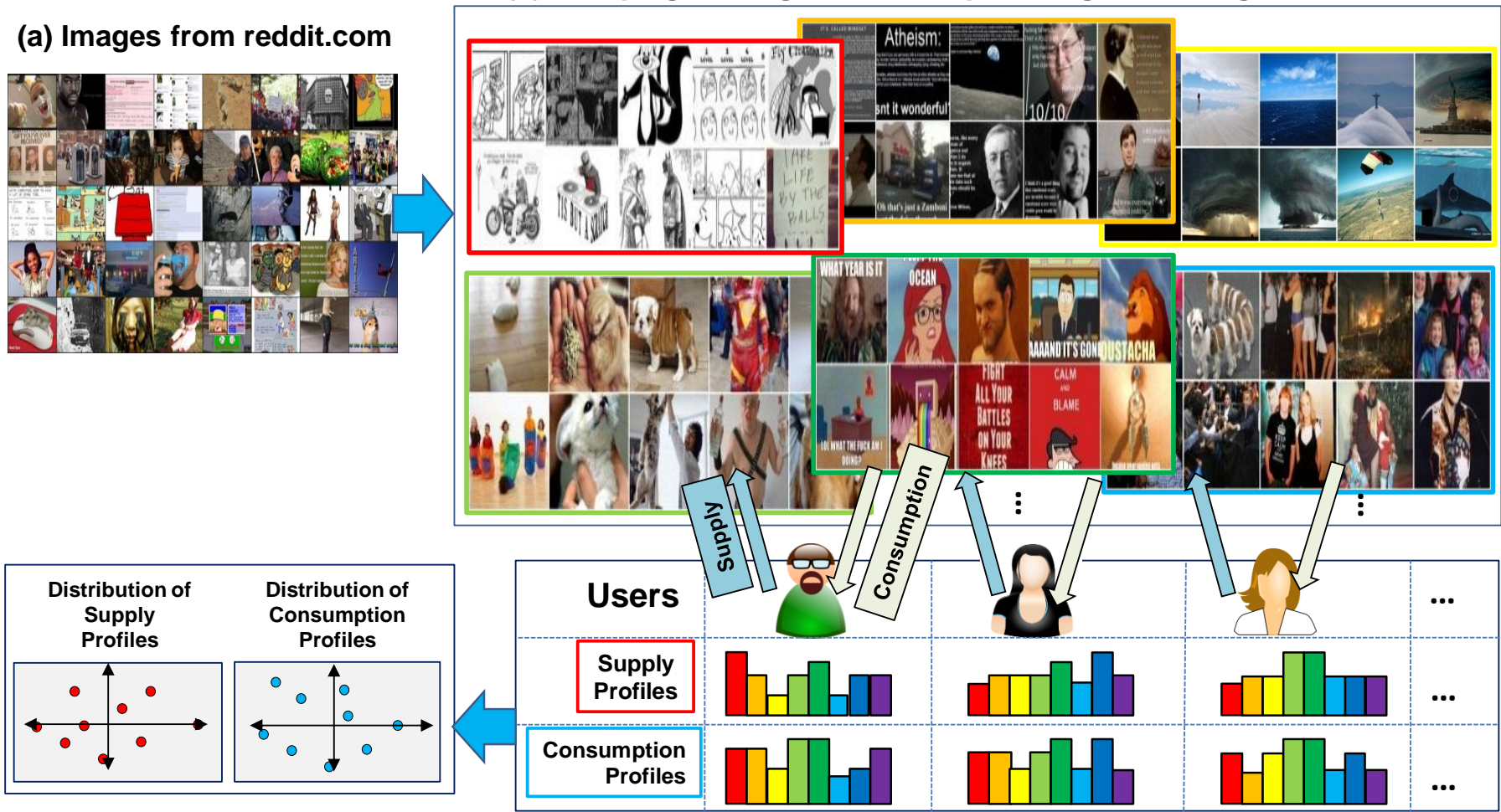

(d) Analysis of Profile Distributions

(c) Individual Profiles as Bag of Words of Image Categories

Fig. 1. Illustration of overall approach. (a) Randomly sampled images from Reddit image dataset. (b) Images are grouped into multiple categories based on their content and styles. Different groups are marked in different colors. (c) Individual user's supply and consumption profiles are computed as bag-ofwords representation based on the history of posting and commenting on different image categories. (d) Profiles of multiple users are analyzed to measure similarity and difference between users' two behavioral modes. Every dot represents a user's profile. Users with salient profiles are identified in this space as well.

derstanding. McAuley and Leskovec (2012) use crowd-sourced Flickr image metadata such as tags, captions, collections etc. to annotate new images with semantic labels. Kovashka and Grauman (2013) worked towards improving image search via a personalized visual attribute classifier. Ottoni et al. (2014) used groups and tags for photos on Pinterest. They analyzed and compared user behavior patterns across text (Twitter) and image (Pinterest) based social networks. However, a lot of visual content on social media is shared without any tags or accompaying text. In many cases, user-supplied tags may not directly refer to the semantic content of the photo they are sharing, e.g., a simple comment such as 'Look at this!'. Thus it becomes necessary to analyze the visual content on its own.

To the best of our knowledge, the only work focusing on understanding user behavior patterns by analyzing the visual properties of the photos they share online is $\mathrm{Hu}$ et al. (2014). They first use low-level visual features to obtain an initial clustering of images. The clusters so obtained are then cleaned up and assigned to one of the eight pre-defined categories by manual annotation. The users are then clustered into five types based on the the content of photos they have shared. Similar to them, we too, cluster the images using low-level features to begin with, and use these clusters to characterize user behavior. However, our work presents more detailed analysis beyond Hu et al. (2014). We build two types of profiles for each user, based on the distribution of the images that they share (supply) and engage with (consume). Further, we also study the temporal patterns of time-varying user behaviors and introduce non-parametric statistical approach to detect salient users and trending content.

Existing work on saliency detection for social multimedia services includes Heard et al. (2010) and Altshuler et al. (2013). They formulate the problem as that of detecting network nodes which exhibit abnormality in terms of structural patterns and connectivity. Rather than focusing on network properties, our saliency detection approach aims to detect salient users based on the actual visual content they supply and consume.

\section{Personalized Image Economy: Models and Analysis}

In this section, we introduce our framework to characterize users' supply and consumption profiles. We also present our findings which showcase the difference between users' two behavioral modes through a statistical analysis of user logs from the Reddit image dataset. The Reddit image dataset consists of a total of 16,736 unique images which have been resubmitted multiple times. There are a total of 132,307 submissions, i.e. each image has been posted 7.9 time on average. Each image post is accompanied with an html page consisting of the communication happening around the post i.e. upvotes and downvotes for the post, the community to which the image was posted, time at which the post was made, comment threads from various users, upvotes and downvotes for the comments etc. 

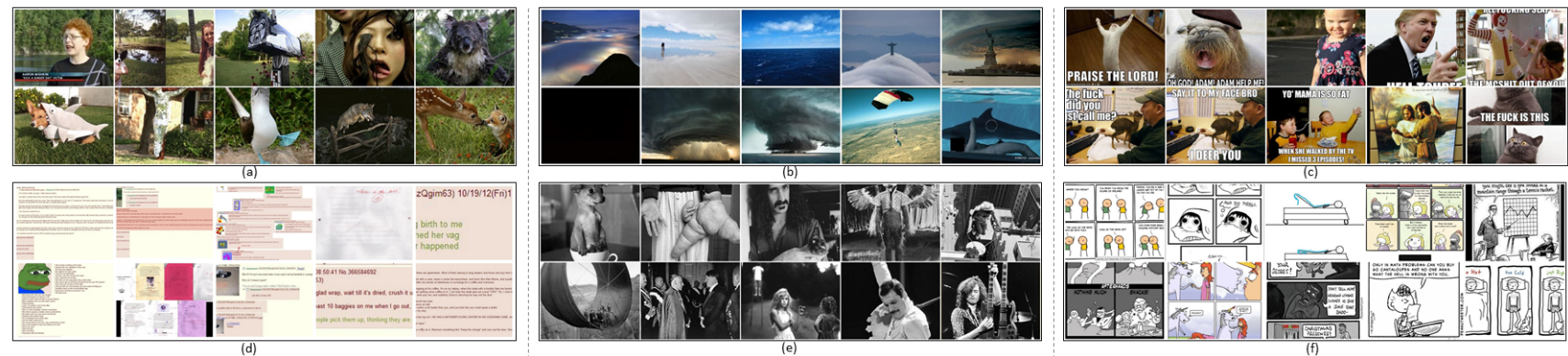

Fig. 2. Clustering of images into recognizable and semantically consistent groups. Randomly selected images from the six sample clusters (out of total 250 ) are shown. (a) Activities in greenery nature, (b) Natural scenes with blue color tone, (c) Memes (images with embedded caption), (d) Historic photos in black \& white (e) Text clips (f) Comic strips.

An overview of our approach is illustrated in Fig. 1. First, the set of images in the Reddit image dataset, some samples from which are shown in Fig. 1(a), are automatically grouped into different image categories based on their visual features. Fig. 1(b) shows a set of sample categories learned during the grouping process described in Sec. 3.1. It can be observed that while the nature of images shared on social forums tends to be extremely diverse, they can still be grouped into fairly coherent categories based on their content and styles. For example, the different groups shown in Fig. 1(b), while noisy, show consistent visual content such as cartoons, faces with text, scenery, animals, cartoons with text, and group of people.

Each user's supply and consumption profiles are computed as the distribution across the learned categories, effectively capturing the image content and styles the user prefers to supply and consume. Fig. 1(c) shows nominal profiles of some users as normalized histograms, where the heights of different color bars represent the frequency of the user's supply or consumption of images from the corresponding image category. For example, the middle user in Fig. 1(c) supplies and consumes images from the cluster marked in dark blue color relatively more often than other users. Based on this process, each user's profile can be represented as two vectors, for supply and consumption respectively, which can then be compared with each other and those of other users as well.

Finally, this section provides a novel statistical analysis on the similarity and difference between users' two behavioral modes. Fig. 1(d) shows two scatter plots, one for supply and another for consumption, where every node represents a user's profile vector. Note that while these nominal illustrations show user profiles in $2 \mathrm{D}$ space, the actual analysis is conducted in $C$ dimensional space in this work, where $C$ is the total number of image categories.

In the following subsections, after more details about automatic image categorization and user profile computation are described in Sec 3.1 and Sec 3.2, Sec. 3.3 presents our methodologies and statistical analysis results about the characteristics of user profiles. Sec. 4 further presents our approaches to identify users with salient profiles in these profile spaces.

\subsection{Learning Grouping of Images by Content and Styles}

The grouping of images used in this work is automatically learned using an unsupervised clustering technique. Visual features computed from images are used as underlying representation. Images are then clustered into $C$ groups using standard
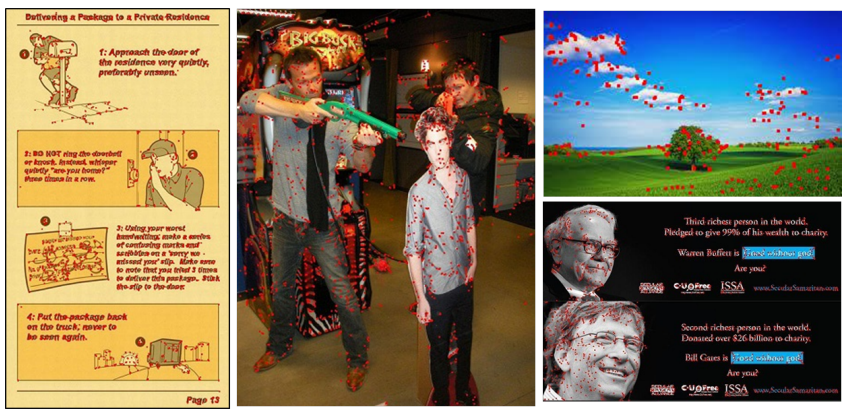

Fig. 3. Illustration of sparse features. In this work, bag-of-words representations of sparse colorSIFT features are used to characterize images. Corner points are detected by a Harris-Laplace corner point detector, which are shown on example images as red dots. Then, colorSIFT feature descriptors are only extracted from these sparse corners.

$\mathrm{K}$-means clustering. Here, $C$ is a pre-specified parameter. In this work, multiple values for $C$ have been tried to capture diverse groups of images at different granularities, including $50,100,250,500$. While most of our analysis presented in the following sections is relatively insensitive to the value of $C$, different parameters do show slight variations, and we will show such variations as feasible to provide more insight. In the rest of the paper, each cluster center will be denoted as $c_{i}$ and the whole set as $\left\{c_{i} \mid 1 \leq i \leq C\right\}$.

Among the diverse image features we explored, we found that visual features which capture both color and structural properties provide the most satisfying grouping results in terms of coherency of content and styles within each group. In particular, we used color-SIFT extraction using van de Sande et al. (2010). For a given image, features were computed on sparse interest points detected on the image using Harris-Laplace corner point detector. The use of sparse features allows our representations to capture more salient foreground content, while being able to ignore largely uniform background which appears frequently in images shared on social forums, e.g. images containing text, and comic strips. Fig. 3 shows sample images with the detected sparse interest points overlaid on top of them. This set of locally computed raw color-SIFT features for each image are then quantized into a bag-of-words $(\mathrm{BoW})$ representation using a codebook size $D$, thus resulting in a $D$-dimensional feature vector. For our experiments in this work, $D=1024$ was used as codebook size, and the image feature vectors were $L_{1}$ normalized. In the rest of the paper, the image feature vector will be denoted as $x_{j}$ for the $j$-th image.

One of our finding is that, despite its simplicity, clustering images based on low-level sparse colorSIFT features provides 

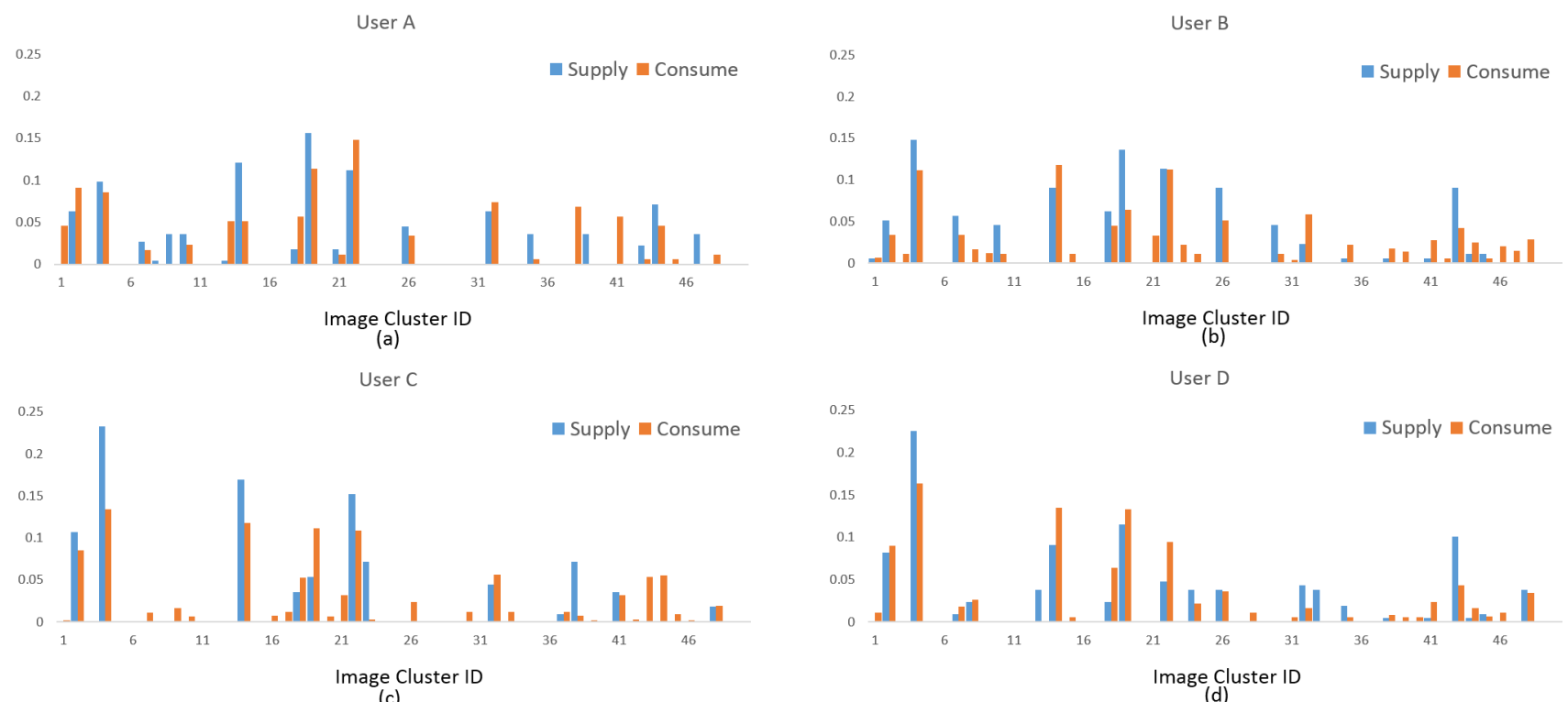

Fig. 4. Visualization of supply and consumption profiles from four randomly selected users when 50 image clusters are used, i.e., $C=50$. It can be observed that while there tends to be correlation between supply and consumption profiles, certain image clusters exhibit fairly different profiles patterns between supply and consumption patterns of a same user.

surprisingly good image clusters, which correlate well with human semantics. In Fig. 2, we show example images from the clusters obtained using $C=250$. A set of randomly selected images from each of these clusters are shown, ten per cluster. Different image categories such as comics, historic images in black and white style, and outdoor scenes were obtained, automatically from a large collection of significantly diverse image set. Although a small subset of image samples shown in Fig. 2 are questionable in terms of their cluster membership and semantic coherence, the grouping results are reasonable and deemed to be sufficient to characterize the image content users engage with, showcasing the effectiveness of the incorporated visual feature.

\subsection{Characterization of User Profiles: Supply $\mathcal{E}$ Consumption}

In this work, we propose a novel approach to characterize a user's supply and consumption profiles as a distribution of image categories with which the user interacts. Based on the $C$ number of learned image categories, every profile is computed as an $L 1$-normalized $C$-dimensional vector, denoted as $y_{k}$ for the $k$-th user. Note that we omit an indicator for supply or consumption in this notation for brevity.

In further detail, each profile $y_{k}$ is a BoW representation which captures the relative frequency with which the corresponding user accesses particular image clusters. To compute these profiles, the log data which records the uploader and commenter information is used. In Reddit dataset, this information is provided as image metadata. In the rest of the paper, the set of image indices $\{j\}$ accessed by the $k$-th user are denoted as a set $\{j \mid k\}$. When metadata indicates that a user engaged with the $j$-th image, either via supply or consumption, its feature vector $x_{j}$ is compared with the $C$ cluster centers. A $C$-dimensional soft quantization vector $x_{j}^{\prime}$ is then computed. Note that $x_{j}^{\prime}$ is $L_{1}$-normalized, i.e., $\left|x_{j}^{\prime}\right|_{1}=1$. Soft quantization is important to avoid unnecessarily harsh boundary effect and produce a more generalizable characterization which is less sensitive to the size $C$ of the clusters used. Essentially, soft quantization represents each image as a weighted mixture of $C$ image categories, where higher weights indicate more similarity. For the experiments reported in this work, we used the soft quantization scheme proposed in Jiang et al. (2007). Finally, each user's profile is computed by averaging the soft quantization vectors over all the images they engaged with, as follows: $y_{k}=\sum_{\{j \mid k\}} x_{j}^{\prime} /|\{j \mid k\}|$ where $\left|y_{k}\right|_{1}=1$. Fig. 4 shows examples of resulting supply and consumption profiles of four randomly sampled users, when $C=50$ image clusters are used. It can be observed that while there tends to be correlation between supply and consumption profiles, certain image clusters exhibit fairly different profiles patterns between supply and consumption patterns of a same user.

\subsection{What does Personalized Image Economy Look Like?}

In this section, we present our findings which are obtained by analyzing the supply and consumption profiles computed from user logs and image data in Reddit dataset.

First, we analyze how the user activities are distributed between consumption and supply, which is visualized in Fig. 5. Here, each point corresponds to a unique user, and the horizontal and vertical coordinates correspond to the number of 'unique' images consumed or supplied by each user, respectively. To improve visibility of details in densely populated areas, log-scale is used for both the axes. By 'unique' images, we mean that when a user has commented multiple times across repeated postings of an identical image, it is counted as only one consumption. Re-posting of popular trending images by multiple users is fairly common in online social forums, and a same user may comment on multiple such postings. Our approach reduces biases introduced by such patterns. Similarly, even if a particular user has posted the same image multiple times, it will count as only one supply. In particular, Fig. 5 includes 


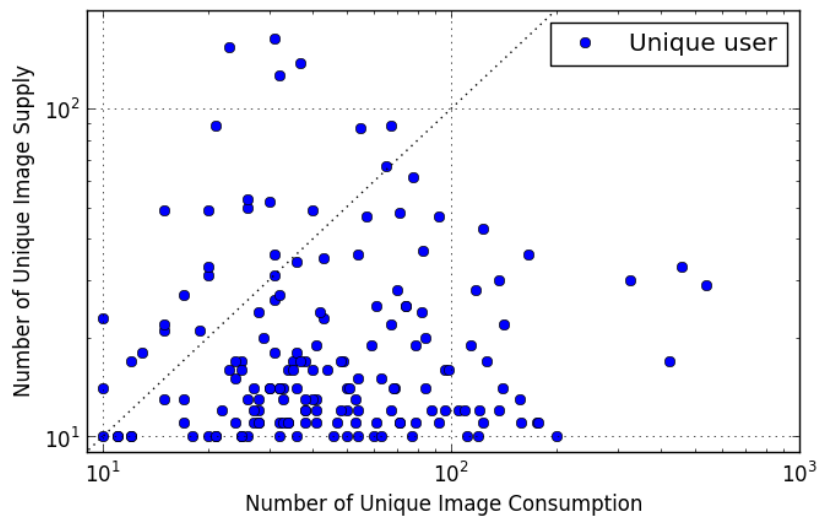

Fig. 5. Activities across users. Each point is a unique user. The horizontal and vertical coordinates correspond to the number of 'unique' images consumed or supplied by each user respectively. Log-scale is used on both axes.

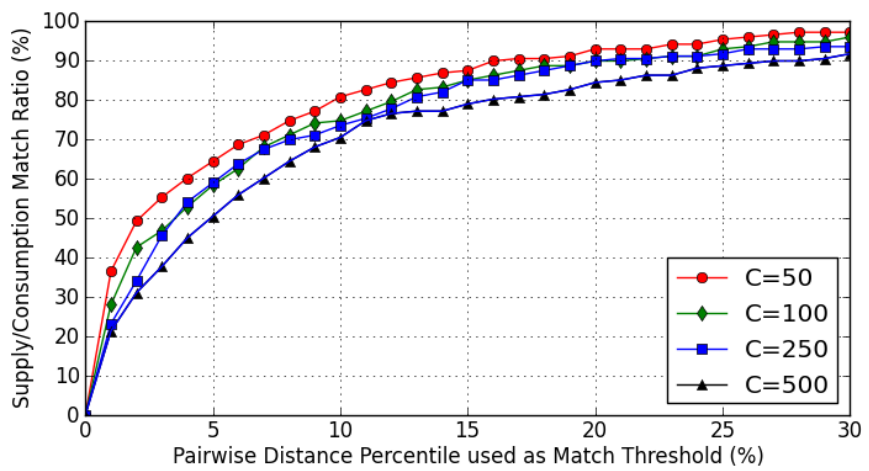

Fig. 6. Ratio of users with similar supply/consumption profiles as a function of varying threshold levels. The match threshold is varying as a function of percentile values within a distribution of all pairwise distances across users. At a relatively large threshold at the 5-th percentile level, as much as $\sim 40 \%$ of users exhibit fairly different supply and consumption behavior.

only users who consumed and supplied at least 10 unique images each in both the modes. There is a total of 165 such users in the dataset, effectively filtering out a large number of users with very low amount of activity in either mode.

An interesting pattern observed from Fig. 5 is the wide variation in the ratio between supply and consumption across users, and the unexpectedly high ratio of users above 1:1 ratio who act more as suppliers than as consumers. Basically, the number of points under 1:1 diagonal line indicates users with heavier 'consumption', and vice versa. While it is generally expected that most users will act as consumers rather than suppliers, actually, a substantial number of users, as much as $~ 15 \%$, show up above the diagonal, and act as committed suppliers who provide unique contents to online social forums.

Further, we have analyzed the similarity and difference between supply and consumption profiles for individual users, and found that significant ratio of users exhibit mismatched profile patterns - i.e., the images many people supply are fairly different from what they consume. We believe this is the first scientific analysis which reports such a finding about images shared on social forums.

For our analysis on profile matching, we have used supply and consumption profiles of the 165 users who supplied and consumed at least 10 unique images, which is the same set of users that appear in Fig. 5. In the rest of the paper, the $k$-th user's supply and consumption profiles are denoted as $y_{k, s}$ and $y_{k, c}$ respectively. Our methodology to determine whether two profiles match is to check whether the two profiles are within a certain match threshold distance $t_{p}$, i.e., $\left\|y_{k, s}-y_{k, c}\right\|_{2}<t_{p}$. Then, the fraction of users whose profiles match can be computed by counting users that satisfy such condition, divided by the total number of users. Fig. 6 shows our analysis where the horizontal axis corresponds to the radius, growing from smaller to larger values, and the vertical axis is the ratio of users with matched profiles. In particular, the match threshold $t_{p}$ is varying as a function of the $p$-th percentile within the distribution of all pairwise distances between users. In detail, the $p$-th percentile match threshold is defined as follows:

$$
\begin{aligned}
A & =\text { sorted array }\left\{\forall_{k \neq k^{\prime}} d_{k, k^{\prime}} \mid d_{k, k^{\prime}}=\left\|y_{k}-y_{k^{\prime}}\right\|_{2}\right\} \\
t_{p} & =A[|A| *(p / 100)]
\end{aligned}
$$

where $A$ is a sorted array (in increasing order) of all the pairwise distances between users' supply profiles collected across users, and $|A|$ indicates the size of the array.

Then, $t_{p}$ is obtained as the $p$-th percentile value from the array $A$. For example, $t_{5}$ is a threshold that corresponds to the top 5 percentile within such distribution. An intuitive interpretation for $t_{p}$ is that, when it is used as a threshold, for any reference user, approximately $p$ percent of the rest of the users are within such radius, on average. Accordingly, our methodology assumes that two profiles do not match at the $p$-th percentile level if the consumption profile of a user is not within the $p$ percent neighborhood of the corresponding supply profile. To provide additional insight regarding the sensitivity of our analysis with respect to the total number of image clusters $C$, Fig. 6 shows analysis for multiple values of $C$. We looked at whether user supply consumption patterns differed when we move from coarse $(C=50)$ to finer $(C=500)$ level of image clustering. Use of soft assignment across image clusters ensures the patterns we see do not suffer due to over-clustering. Note that the average difference in the match ratio (y-axis) across different image cluster sizes used is approximately $15 \%$. Other than that, results across different values of $C$ show similar progression patterns.

An interesting finding from the analysis shown in Fig. 6 is that, at the 5-th percentile threshold $t_{5}$, only $\sim 60 \%$ users show matched profiles (for $C=100$ and 250), while the remaining $40 \%$ of users exhibit mismatch patterns. It is worth noting that even the threshold $t_{5}$ is fairly large in the sense that it will generally contain about $5 \%$ of the entire social forum users within its radius, which is a fairly large number. This finding contradicts a baseline hypothesis that users are likely to post and comment on similar image categories, and suggests that personalization of social multimedia services needs to be optimized from both angles, rather than pursuing a single model for each user. For example, it can be conjectured that bi-directional social network modeling will work more accurately to predict the propagation of popular images across users, rather than undirected network model alternatives. 


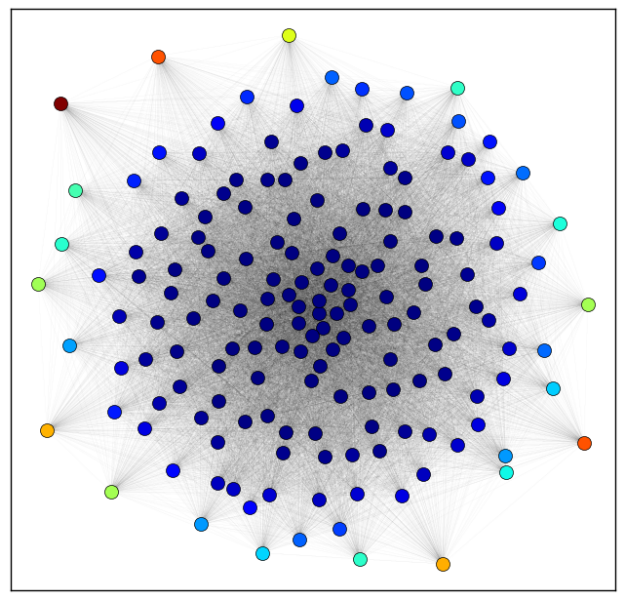

(a)

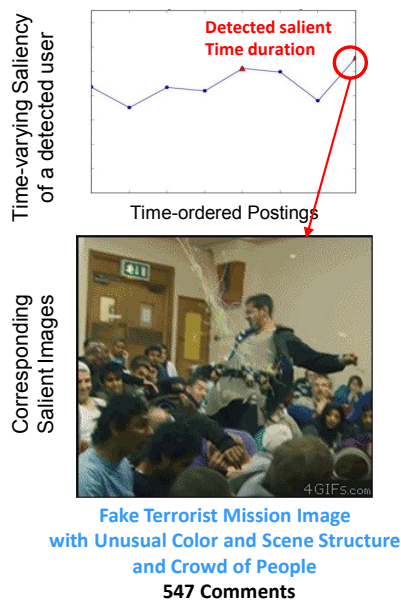

$\mathbf{5 4 7}$ Comments
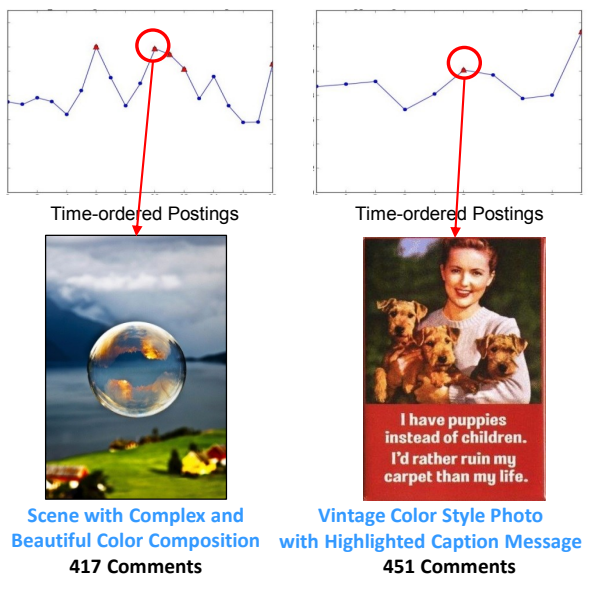

(b)

Fig. 7. (a) Detected salient users (bright colors) with respect to the whole user group. Salient users are detected using kernel density approximation technique from high-dimensional profile space. The detected users appear at boundaries which showcase the usefulness of the proposed approach. (b) Illustration of our approach to identify salient key images. (b,top) Examples of time-varying saliency from three salient users where detected salient durations are marked by red triangles. (b,bottom) Example images sampled from detected salient durations, which include fake terrorist act, supernatural scene, and vintage-style photo with controversial caption. Images show unique visual characteristics, which increases their saliency, and receive large amount of engagement.

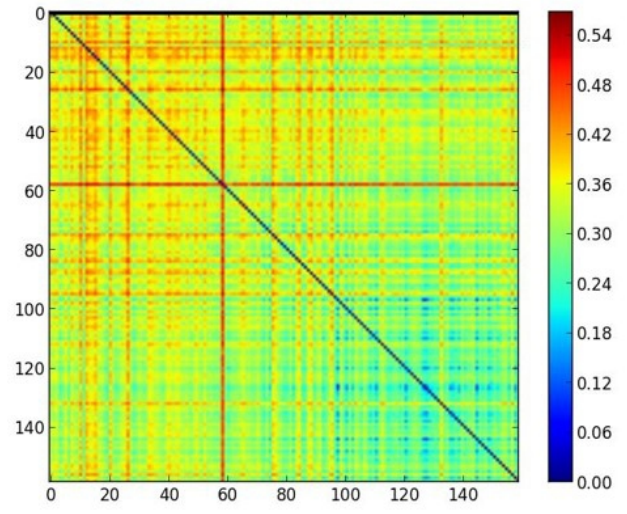

Fig. 8. Pairwise distance among 150 supply profiles from users who posted(supplied) more than 30 posts. Their user profiles are computed using 250 image clusters. The value of the $(i, j)$ element of the above matrix indicates pairwise distance between the $i$-th and $j$-th users.

\section{Detecting Salient Users and Images}

In this section, we introduce our approach to identify salient users along with the key images which substantially contributed to user saliency. In particular, we focus on identifying salient 'suppliers' and images they post, because supply is arguably a more important contributing factor towards a success or a failure of a social multimedia service. A novel aspect of our approach is that user saliency is measured based on the visual content they supply, i.e., their supply profiles, while most existing work such as Heard et al. (2010); Altshuler et al. (2013) addresses this problem by analyzing abnormal structural patterns in social networks such as properties of friendship connectivity. The saliency in supply profiles is illustrated in Fig. 8, which shows pair-wise distances between supply profiles of 150 randomly sampled users. For this example, we only considered users who posted more than 30 images. Then, a supply profile is featurized as a BoW representation over 250 image clusters.
We computed their pairwise $L 2$ distances and visualized them in a matrix form, where the $(i, j)$ value indicates distance between the $i$-th and $j$-th supply profiles. We can clearly observe a salient supply profile at row 59 (column 59), which shows fairly large distance from other profiles.

In addition, we also show that user saliency may be timevarying - a user who exhibits regular behavior in general may still show salient patterns during certain time intervals, and by detecting such intervals, we can identify key images that contribute significantly to make a user salient.

\subsection{Detecting Salient Users using Kernel Density Estimation}

Social forum users are diverse in nature. Thus the distribution of user profiles tends to exhibit numerous local modes. By definition, a salient user exhibits a unique profile, which is likely to appear in low-density regions. To detect salient users, we propose a detection method based on non-parametric kernel density estimation techniques Epanechnikov (1969). We estimate the likelihood of a user's profile as a weighted sum of its similarity towards the rest of the users. In detail, for the $k$-th user profile $y_{k}$, the likelihood $p\left(y_{k}\right)$ is defined as:

$$
p\left(y_{k}\right)=\frac{1}{|\{i \backslash k\}|} \sum_{\{i \backslash k\}} \alpha \exp \left\{-\frac{\left(y_{k}-y_{i}\right)^{2}}{\sigma^{2}}\right\},
$$

where $\{i \backslash k\}$ indicates the set of all indices except $k$ and $\alpha$ and $\sigma$ are parameters of Gaussian kernel function, e.g., $\sigma=0.1$. Low-likelihood corresponds to high saliency. Accordingly, we define saliency $s\left(y_{k}\right)$ to be an inverse exponential function of likelihood as follows: $s\left(y_{k}\right)=e^{-p\left(y_{k}\right)}$.

The effectiveness of the proposed approach can be observed from the visualization of detected salient users (bright color) along with the rest of the users (dark color) shown in Fig. 7(a). Each node corresponds to a user and the length of the edges between users correspond to the distance between user profiles. 
For this visualization, the coordinates of user nodes are computed using the graph layout technique in Dwyer (2009) which incorporates edge distance constraints. It can be observed that users with salient profiles identified by the proposed method appear mostly at the boundary as expected ${ }^{2}$.

\subsection{Detecting Key Images from Salient Users}

Once salient users have been identified, we identify key images which contributed the most towards saliency. We compute the time-varying saliency of the detected users from timeordered images based on their posting history. A time-window was used to compute the saliency at every few posts, effectively creating detailed snapshots of temporal user profiles using the identical technique as Sec. 3.2. For example, Fig. 7(b, top) shows the varying saliency of three example salient users (yaxis) as a function of time (x-axis). For these results, we used a time window of size 10 , and a stride size of 5 images.

In Fig. 7(b, top), it can be observed that the saliency of users tends to vary substantially across posts. Subsequently, using thresholds, certain durations are detected to be significantly salient, which are marked as red triangles on the graph (best viewable in zoom mode) in Fig. 7(b).

For a qualitative assessment, we sampled images from each flagged duration, as shown at the bottom of Fig. 7(b). These example images show a fake terrorist scene, a super-natural scenery, and a vintage style image with a highlighted caption which contain controversial argument about family value. Overall, these images showcase that the proposed method can identify images with interesting and unusual visual characteristics. We have also observed that such images tend to be consumed heavily by diverse users, as can be observed from the number of comments each image received.

We believe that our approach of detecting salient users and images can identify useful information which can be used to improve the quality of social multimedia services, such as detecting and promoting viral contents early, or down-weighting postings deemed to be unfit for the social communities. Automatic approaches to perform such follow-up actions have not been explored, but remain to be addressed in our future work.

\section{Conclusion}

In this work, we have addressed the problem of characterizing individual users' supply and consumption profiles with respect to extremely diverse images on social forums. Our analysis shows that there are a significant number of users who show fairly different behavior in terms of the images they supply and consume, which suggests that personalization of social multimedia services needs to be optimized from both the angles. Additionally, we have proposed an approach to identify salient users and key images they engage with based on their temporal profile. Our experimental results demonstrate the

\footnotetext{
${ }^{2}$ Note that the illustration in Fig. 7(a) is not optimized to show the multimodal nature of full distribution on 2D. A PCA-based 2D projection is an approach that is applicable, however, we found that the diversity of user profiles are not characterized by 2 dimensions effectively.
}

usefulness of the proposed approaches.

Acknowledgement: Research Projects Agency (DARPA) under Contract No. HR0011-10-C-0112. Any opinions, findings and conclusions or recommendations expressed in this material are those of the authors and do not necessarily reflect the views of DARPA or the U.S. Government. The views and conclusions contained in this document are those of the authors and should not be interpreted as representing the official policies, either expressly or implied, of the DARPA or the U.S. Government. All authors were with Kitware when this work was conducted.

\section{References}

Altshuler, Y., Fire, M., Shmueli, E., Elovici, Y., Bruckstein, A., Pentland, A.S., Lazer, D., 2013. Detecting anomalous behaviors using structural properties of social networks, in: Social Computing, Behavioral-Cultural Modeling and Prediction. Springer, pp. 433-440.

Can, E.F., Oktay, H., Manmatha, R., 2013. Predicting retweet count using visual cues, in: Proceedings of ACM International Conference on information and knowledge management (CIKM).

Cha, M., Kwak, H., Rodriguez, P., Ahn, Y.Y., Moon, S., 2009a. I Tube, You Tube, Everybody Tubes: Analyzing the Worlds Largest User Generated Content Video System, in: Usenix/ACM SIGCOMM Internet Measurement Conference (IMC).

Cha, M., Mislove, A., Gummadi, K., 2009b. A Measurement-driven Analysis of Information Propagation in the Flickr Social Network, in: International World Wide Web Conference (WWW).

Danescu-Niculescu-Mizil, C., West, R., Jurafsky, D., Leskovec, J., Potts, C., 2013. No Country for Old Members: User Lifecycle and Linguistic Change in Online Communities, in: International World Wide Web Conference (WWW).

Dwyer, T., 2009. Scalable, versatile and simple constrained graph layout, in: Computer Graphics Forum, Wiley Online Library. pp. 991-998.

Epanechnikov, V.A., 1969. Non-parametric estimation of a multivariate probability density. Theory of Probability and Its Applications 14, 153-158.

Heard, N.A., Weston, D.J., Platanioti, K., Hand, D.J., 2010. Bayesian anomaly detection methods for social networks. The Annals of Applied Statistics 4, 645-662.

Hong, L., Dan, O., Davison, B.D., 2011. RT to Win! Predicting Message Propagation in Twitter, in: International World Wide Web Conference (WWW).

$\mathrm{Hu}$, Y., Manikonda, L., , Kambhampati, S., 2014. What we instagram: A first analysis of instagram photo content and user types, in: AAAI International Conference on Weblogs and Social Media (ICWSM).

Jiang, Y.G., Ngo, C.W., Yang, J., 2007. Towards optimal bag-of-features for object categorization and semantic video retrieval, in: Proceedings of the ACM international conference on Image and video retrieval (CIVR), ACM.

Kovashka, A., Grauman, K., 2013. Attribute Adaptation for Personalized Image Search, in: International Conference on Computer Vision (ICCV).

Lakkaraju, H., McAuley, J., Leskovec, J., 2013. Whats in a name? Understanding the Interplay between Titles, Content, and Communities in Social Media, in: AAAI International Conference on Weblogs and Social Media (ICWSM).

McAuley, J., Leskovec, J., 2012. Image Lablineg on a Network: Using SocialNetwork Metadata for Image Classification, in: European Conference on Computer Vision (ECCV).

Ottoni, R., de Las Casas, D., Pesce, J.P., Jr, W.M., C.Wilson, Mislove, A., Almeida, V., 2014. Of Pins and Tweets: Investigating how users behave across image- and text-based social networks, in: AAAI International Conference on Weblogs and Social Media (ICWSM).

Petrovic, S., Osborne, M., Lavrenko, V., 2011. RT to Win! Predicting Message Propagation in Twitter, in: International AAAI Conference on Weblogs and Social Media (ICWSM).

van de Sande, K.E.A., Gevers, T., Snoek, C.G.M., 2010. Evaluating Color Descriptors for Object and Scene Recognition 32, 1582-1596.

Sundaram, H., Xie, L., De Choudhury, M., Lin, Y.R., Natsev, A., 2012. Multimedia semantics: Interactions between content and community. Proceedings of the IEEE 100, 2737-2758.

Uysal, I., Croft, W.B., 2011. User Oriented Tweet Ranking: A Filtering Approach to Microblogs, in: Proceedings of ACM International Conference on information and knowledge management (CIKM). 